INPLASY



To cite: Liu et al. The effect of acupoint application of traditional Chinese medicine for the treatment of chronic obstructive pulmonary disease: a protocol for systematic review and metaanalysis. Inplasy protocol 202090023. doi:

10.37766/inplasy2020.9.0023

Received: 06 September 2020

Published: 06 September 2020

Corresponding author:

Yefang Liu

Liuyefang@cdutcm.edu.cn

Author Affiliation:

Affiliated Hospital of Chengdu University of TCM (West

District), Chengdu Pidu District

Hospital of TCM, Chengdu,

Sichuan, China

\section{Support: SCATCM:2016C067.}

Review Stage at time of this submission: Formal screening of search results against eligibility criteria.

Conflicts of interest:

The authors have declared that no competing interest exists.
The effect of acupoint application of traditional Chinese medicine for the treatment of chronic obstructive pulmonary disease: a protocol for systematic review and meta-analysis

Liu, YF1; Li, Y2; Zeng, SQ3; Su, Y4; Zhuang, GT5; Liu, XY6; Chen, L7; Feng, QS 8 .

Review question / Objective: Is acupoint application of traditional Chinese medicine efficacy and safety for COPD? Condition being studied: Chronic obstructive pulmonary disease (COPD) is a public threat, leading to progressive physical activity and poor quality of life. Although modern medicine has excellent achievement of COPD, the recurrence rate of stable COPD and the mortality of acute exacerbation COPD remain high. As one of the external therapy of traditional Chinese medicine (TCM), acupoint application has been treated COPD in China for a long time. Nevertheless, study evaluating the effect of acupoint application for COPD could not satisfy needs for clinic.

INPLASY registration number: This protocol was registered with the International Platform of Registered Systematic Review and Meta-Analysis Protocols (INPLASY) on 06 September 2020 and was last updated on 06 September 2020 (registration number INPLASY202090023).

\title{
INTRODUCTION
}

Review question / Objective: Is acupoint application of traditional Chinese medicine efficacy and safety for COPD?

Condition being studied: Chronic obstructive pulmonary disease (COPD) is a public threat, leading to progressive 
physical activity and poor quality of life. Although modern medicine has excellent achievement of COPD, the recurrence rate of stable COPD and the mortality of acute exacerbation COPD remain high. As one of the external therapy of traditional Chinese medicine (TCM), acupoint application has been treated COPD in China for a long time. Nevertheless, study evaluating the effect of acupoint application for COPD could not satisfy needs for clinic.

\section{METHODS}

Search strategy: Qualified studies were extracted through literature search using Web of Science, Pub Med, EMBASE, Cochrane Library, China National Knowledge Infrastructure (CNKI), Wan Fang, Chinese Scientific Journals Database (VIP) and Chinese Biomedical Database (CBM) from inception to March 2020. And we also used references from previously published systematic reviews to manually search for relevant research. The following key search terms, including relative medical subject heading (Mesh) and free text term will be retrieved : "Chinese herbal medicine" or "traditional Chinese medicine" or "TCM" or "acupoint application" or "Chinese acupoint application" or "Herbal medicine point application" or "Chronic obstructive pulmonary disease" or "COPD" or "acute exacerbation COPD" or "AECOPD" or "stable COPD".

Participant or population: Diagnostic criteria: Patients who were diagnosed with COPD. Exclusion of severe cardiovascular, cerebrovascular diseases and other complications. Exclusion of patients who are allergic to Chinese medicine application, or patients with skin rupture, hypersensitivity or scar constitution.

Intervention: Experimental group is conventional therapy combined with acupoint application regardless of herbal regimen, acupoints selected, patching time are eligible for inclusion. Control group is conventional therapy combined with placebo or not.
Comparator: Experimental group is conventional therapy combined with acupoint application regardless of herbal regimen, acupoints selected, patching time are eligible for inclusion. Control group is conventional therapy combined with placebo or not.

Study designs to be included: Inclusion: Whether they are blind or not, only clinical randomized controlled trial (RCT) articles will be included of Chinese and English language. Exclusion: Animal experimental study and quasi-randomized trials will be excluded.

Eligibility criteria: Whether they are blind or not, only clinical randomized controlled trial (RCT) articles will be included of Chinese and English language. Patients who were diagnosed with COPD are study participants. Experimental group is conventional therapy combined with acupoint application regardless of herbal regimen, acupoints selected, patching time are eligible for inclusion. Control group is conventional therapy combined with placebo or not.

Information sources: Qualified studies were extracted through literature search using Web of Science, Pub Med, EMBASE, Cochrane Library, China National Knowledge Infrastructure (CNKI), Wan Fang, Chinese Scientific Journals Database (VIP) and Chinese Biomedical Database (CBM) from inception to March 2020. And we also used references from previously published systematic reviews to manually search for relevant research. The following key search terms, including relative medical subject heading (Mesh) and free text term will be retrieved : "Chinese herbal medicine" or "traditional Chinese medicine" or "TCM" or "acupoint application" or "Chinese acupoint application" or "Herbal medicine point application" or "Chronic obstructive pulmonary disease" or "COPD" or "acute exacerbation COPD" or "AECOPD" or "stable COPD". 
Main outcome(s): Obvious efficiency, effective rate and clinical control rate and lung function are primary outcomes.

Additional outcome(s): Assessment scales and adverse reactions are additional outcomes.

Quality assessment / Risk of bias analysis: The study will be assessed through Cochrane Handbook for Systematic Reviews of Interventions. These indicators is categorized different risk of bias (including low, high and unclear) if needed. The different opinion of two reviewers will be resolved by consultation with the corresponding authors.

Strategy of data synthesis: We will analyses results by Review Manager software. For continuous outcomes, mean difference (MD) with $95 \%$ confidence intervals (Cist) will be usage. For dichotomous outcomes, risk ratio (RR) will be adopted. ${ }^{2}$ statistics will be used to assess heterogeneity. If no significant heterogeneity in the data $(P>.05$ and $I^{2}<50 \%$ ), a fixed-effect (FE) model will be performed. If significant heterogeneity is found $\left(P<.05\right.$ and $\left.I^{2}>50 \%\right)$, a randomeffects (RE) model will be conducted.

Subgroup analysis: Clinical stage of acute exacerbation and stable will be carried out as one pre-specified subgroup analyses if possible.

Sensibility analysis: If heterogeneity is significantly different, then sensitivity analysis method will be accepted for exploring it, based on Corresponding clinical parameters.

Language: Chinese and English.

Country(ies) involved: China.

Keywords: Acupoint application, chronic obstructive pulmonary disease, protocol, systematic review.

Contributions of each author:

Author 1 - Yefang Liu.

Author 2 - Yu Li.

Author 3 - Shaoqian Zeng.
Author 4 - Yue Su.

Author 5 - Guangtong Zhuang.

Author 6 - Xiyang Liu.

Author 7 - Lin Chen.

Author 8 - Quansheng Feng. 